\title{
Carrier density dependence of $1 / f$ noise in graphene explained as a result of the interplay between band-structure and inhomogeneities
}

Received: date / Accepted: date

\begin{abstract}
We present a model for $1 / f$ noise in graphene based on an analysis of the effect of charge trapping and detrapping events on the fluctuations of the number of charge carriers. Inclusion of a Gaussian distribution of fluctuations of the electrostatic potential enables us to reproduce all the various experimentally observed behaviors of the flicker noise power spectral density as a function of carrier density, both for monolayer and bilayer graphene. The key feature of a flicker noise minimum at the Dirac point that appears in bilayer graphene and sometimes also in monolayer graphene is explained in terms of the disappearance, when the number of electrons equals that of holes, of the carrier number fluctuations induced by trapping events. Such a disappearance is analyzed with two different approaches, in order to gain a better understanding of the physical origin of the effect, and to make some considerations about possible analogous phenomena in other semiconductors.
\end{abstract}

Keywords Graphene (Theory) · Disordered systems (Theory) · Current fluctuations

PACS PACS 81.05.ue · PACS 73.50.Td · 73.22.Pr

\section{Introduction}

Flicker noise in graphene based devices has attracted significant interest [1] because of the very peculiar features it exhibits, in comparison with what is observed in more traditional materials. In particular, the behavior of the noise power spectral density as a function of carrier concentration has turned out to be rather puzzling, especially in bilayer graphene, and several authors [2-6] have made an effort to find an explanation for it. The most striking characteristic is that the power spectral density of flicker noise in bilayer graphene, and in a few instances also in monolayer graphene, has a minimum around the Dirac point, where charge concentration also reaches a minimum, while in conductors obeying Hooge's empirical formula [7] the opposite is expected. Attempts have been made to justify the particular dependence of the flicker power spectral density on carrier concentration on the basis of the known presence of electron and hole puddles in the graphene sheet [2], of a supposed variation [3] of the Hooge parameter with gate voltage, which would prevail on the effect of the carrier number decrease, of effects linked to mobility fluctuations [4], of a charge-noise model [5], or of the bandstructure of single layer and bilayer graphene [6]. We make an effort to provide a framework within which a more general and intuitive understanding of flicker noise in graphene can be derived, focusing on two approaches to the analysis of charge carrier statistics in the presence of carrier trapping events. In particular, we consider a first procedure that relies on the evaluation of the change of occupancy in the 2-dimensional electron or hole gas as a result of the potential perturbation due to a trapped charge, and a second procedure that is based on enforcing neutrality and the mass-action law. We show that results are very similar and the most striking feature is that close to the Dirac point trapping of a charge does not lead to

Dipartimento di Ingegneria dell'Informazione, via G. Caruso 16, 56122 Pisa, Italy E-mail: macucci@mercurio.iet.unipi.it 
a variation of the total number of carriers, which leads to a drop of the flicker noise level, unless the effects of disorder prevail.

\section{Current fluctuations}

Our aim is to find an expression for the power spectral density of flicker current noise in graphenebased devices. We assume that flicker noise is the result of charges moving into and out of traps in the proximity of the channel in which the current flows. We also assume that such fluctuations occur on a time scale much longer than that of carrier scattering events (such as phonon scattering), and, in particular, than that of electron-hole thermal generation. Thus the contribution of each elementary area of the device is due to the fluctuation of the local value of the drift current. This can be related to the current at the terminals via the Electrokinematics theorem [9], which is an extension of the RamoShockley theorem $[10,11]$. In particular, if, for the sake of simplicity, we do not enter into the specific details of the device geometry and assume the electric field $E$ to be somewhat constant across the device, we can write the current at the terminals, as long as we are interested just in the low-frequency fluctuations, in the form

$$
I=\frac{1}{L} \int_{A} q \mu n_{c} E d x d y
$$

where $\mu$ is the mobility, $A=W L$ the area of the device, $L$ being its length and $W$ its width. Let us now move on to the evaluation of the fluctuations of the current. We are interested only in the fluctuations due to charges moving into and out of traps, therefore we can consider just the action of the traps. In principle, trapping and detrapping events have an effect not only on the number of carriers available for conduction, but also on the mobility, and on the local electric field. These two latter contributions are often negligible with respect to the former [12], so that the relative current fluctuation for each trap can be written

$$
\frac{\Delta i}{I}=\frac{1}{A} \int_{A} \frac{\Delta n_{c}}{n_{c}} d x d y
$$

where $n_{c}=n+p$, i.e. the total concentration of carriers ( $n$ is the electron concentration and $p$ is the hole concentration), while we also define $n_{n}=n-p$, which, multiplied by the electron charge, gives the total charge density.

Let us now introduce a first method for the determination of $\Delta n_{c}$ resulting from a trapping event. We consider the effect that the perturbation of the electrostatic potential $U$ due to a trapped charge has on the occupancy of the carrier gas in a region corresponding to a few screening lengths. Since $\Delta n_{c}=\left(\partial n_{c} / \partial U\right) \Delta U$ and $\Delta n_{n}=\left(\partial n_{n} / \partial U\right) \Delta U$, Eq. (2) becomes:

$$
\begin{aligned}
\frac{\Delta i}{I} & =\frac{1}{A} \int_{A} \frac{\frac{\partial n_{c}}{\partial U} \Delta U}{n_{c}} d x d y \\
& =\frac{1}{A} \int_{A} \frac{\frac{\partial n_{c}}{\partial U}}{n_{c}} \frac{\Delta n_{n}}{\frac{\partial n_{n}}{\partial U}} d x d y
\end{aligned}
$$

where the integration can be limited to the region $\delta A$, with a radius of a few screening lengths, within which a perturbation of the potential can be observed. With the further approximation of considering $n_{c}$ and the partial derivatives of $n_{c}$ and $n_{n}$ constant within $\delta A$, we can write

$$
\frac{\Delta i}{I}=\frac{1}{A} \frac{a_{c}}{a n_{c}} \int_{\delta A} \Delta n_{n} d x d y
$$

where we have defined $a_{c}=-\partial n_{c} / \partial U$ and $a=-\partial n_{n} / \partial U$. Since, in order to guarantee neutrality, the integral of the variation $\Delta n_{n}$ of $n_{n}$ over the screening region $\delta A$ must equal the opposite of the variation $\Delta \chi$ of the trap occupancy (where $\chi$ is defined as 1 when the trap is occupied by an electron and as 0 when the trap is empty), we can write [12]

$$
\frac{\Delta i}{I}=-\frac{1}{A}\left(\frac{a_{c}}{a n_{c}}\right) \Delta \chi
$$


The values of $a_{c}$ and $a$ depend on the position of the Fermi level, and can be obtained from the expressions of $n_{c}$ and $n_{n}$, respectively, which can in turn be derived from those of $n$ and $p$, computed [12] with a proper integration of the product of the density of states times the Fermi function [13], exploiting the dispersion relations of monolayer $[14,15]$ and bilayer [16] graphene.
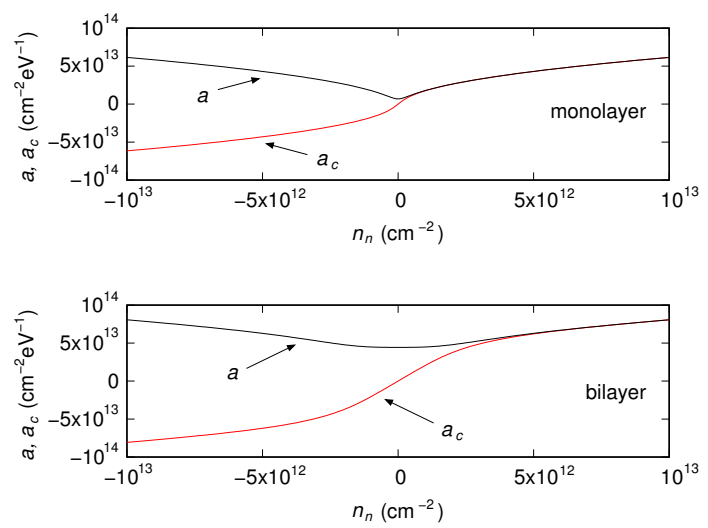

Fig. 1 Behavior of $a_{c}=-\partial n_{c} / \partial U$ and $a=-\partial n_{n} / \partial U$, as a function of $n_{n}=n-p$, for the cases of monolayer (upper panel) and bilayer (lower panel) graphene.

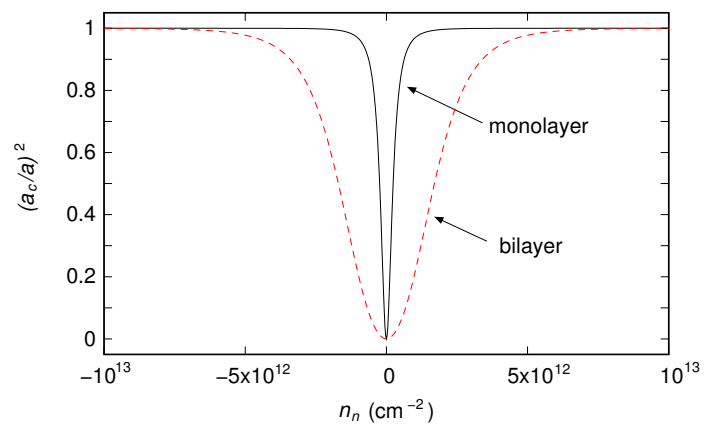

Fig. 2 Behavior of $\left(a_{c} / a\right)^{2}$, as a function of $n_{n}$, for the cases of monolayer and bilayer graphene.

In Fig. 1 and Fig. 2 we report the behavior of $a_{c}$ and $a$, as well as their squared ratio, for the cases of monolayer and bilayer graphene, as a function of $n_{n}$. The most prominent feature is that $a_{c}$ vanishes when the number of electrons equals that of holes (defined as Dirac point in the case of graphene). This involves the important consequence that in perfectly ordered graphene noise due to trapping and detrapping events would disappear at the Dirac point, both in monolayer and in bilayer graphene. We notice that the dependence on $n_{n}$ of the $a_{c} / a$ ratio for monolayer graphene is steeper than that for bilayer graphene: this will have important consequences on the results that we will later obtain including disorder.

Each trap contributes with the fluctuation of its occupancy $\chi$ to the overall noise spectrum. Since $\chi$ is a random telegraph signal, its spectrum is Lorentzian [17] and, as well known, a superposition of Lorentzian spectra associated with traps with properly distributed time constants leads to a $1 / f$ spectrum $[18,19]$

$$
\frac{S}{I^{2}}=\frac{\eta}{A}\left(\frac{a_{c}}{a n_{c}}\right)^{2} \frac{1}{f^{\gamma}},
$$

where $\eta$ is a coefficient depending on the trap density and on the statistics of the traps, and the exponent $\gamma$ is usually 1 (flicker noise). 
We can reach an analogous result with a different approach, which also enables us to understand some further detail of the charge fluctuation process. Let us start again from Eq. (2) and compute the quantity $\Delta n_{c}$ for the case of an electron being trapped (the case of hole trapping is exactly symmetric). When an electron is captured by a trap located in the graphene layer or in its vicinity, the channel remains neutral without the need for charges entering or leaving through the contacts. Therefore, independent of the position of the Fermi level (and therefore of the relative concentration of electrons and holes), the instantaneous variation of the number of carriers is -1 . However, over times that exceed the thermal generation-recombination time constants, the number of each type of free carriers must be consistent with the proper statistics and, in particular, must obey the mass-action law. From electroneutrality we can write that

$$
\Delta N-\Delta P=-1,
$$

where $\Delta N$ and $\Delta P$ are the variations (resulting from the trapping event) of the number $N$ of electrons and $P$ of holes, respectively $\left(N=\int_{A^{\prime}} n d x d y\right.$ and $P=\int_{A^{\prime}} p d x d y$, where $A^{\prime}$ is the total area of the device or, more simply, an area that includes all the perturbations produced by the charge trapping event). Here we have assumed that neutrality was satisfied before the trapping (i.e. the difference between $N$ and $P$ was compensated by the charges on the biasing electrodes) and is satisfied afterwards. From the mass-action law, we can write

$$
(\Delta P+P)(\Delta N+N)=c,
$$

where $c$ is a constant with a value depending on the position of the Fermi level, whose perturbation as a result of the trapping event of an electron we assume to be negligible. Therefore it is also true that $P N=c$ and thus Eq. (8) becomes

$$
P \Delta N+\Delta P N+\Delta P \Delta N=0,
$$

which, using Eq. (7) reads

$$
(\Delta N)^{2}+\Delta N(P+N+1)+N=0,
$$

that we can solve for $\Delta N$. It can be shown that, as long as $N$ and $P$ are much larger than $1, \Delta N$ depends mainly on the ratio of $N$ to $P$ and very weakly on the absolute magnitudes of $N$ and $P$. Thus the size of the actual region of integration $A^{\prime}$ is not relevant.

If electrons are the prevailing type of charge carriers, $\Delta N$ is substantially equal to -1 , since the trapped electron will be screened mainly by other electrons and, overall, the number of mobile charges will differ from that before the trapping event just by one electron. In the opposite limit, of prevailing holes, Eq. (10) will yield a value of 0 for $\Delta N$, which implies $\Delta P=1$. This is consistent with the trapped electron being screened by holes, with an overall increase by 1 of the number of mobile carriers. The most interesting situation is in the Dirac point, where we get $\Delta N=-1 / 2, \Delta P=1 / 2$, which involves symmetric screening of the trapped electron and no variation in the mobile charge.

The values of $n$ and $p$ [and thus of $N$ and $P$, which are needed for the solution of Eq. (10)] can be computed with an integration, as previously explained in the description of the other method, to obtain the fluctuation of $n_{c}$.

We point out that, while in non-degenerate semiconductors the $n p$ product is substantially constant, in graphene (both monolayer and bilayer) it depends on the position of the Fermi level (a clear discussion of this issue for monolayer graphene can be found, for example, in Ref. [20]).

In Fig. 3 we plot the value of the square of the quantity $\Delta N+\Delta P=\int_{A} n_{c} d x d y$ as a function of $n_{n}$ for monolayer (solid curve) and for bilayer (dashed curve) graphene. The square of this quantity can be directly used for the evaluation of the noise power spectral density, and we obtain the equivalent of Eq. (6):

$$
\frac{S}{I^{2}}=\frac{\eta^{\prime}}{A}\left(\frac{\Delta N+\Delta P}{n_{c}}\right)^{2} \frac{1}{f^{\gamma}},
$$

where $\eta^{\prime}$ is a proper coefficient depending on the trap density and on trap statistics.

The behavior of the flicker noise power spectral density as a function of $n_{n}$ from Eqs. (6) and (11) is very similar and is reported, both for monolayer and for bilayer graphene, in Fig. 4 [from Eq. (6)] and in Fig. 5 [from Eq. (11)]. These plots are obtained in the hypothesis of perfectly ordered material, and both of them exhibit a minimum of the noise power spectral density in the Dirac point, which is 


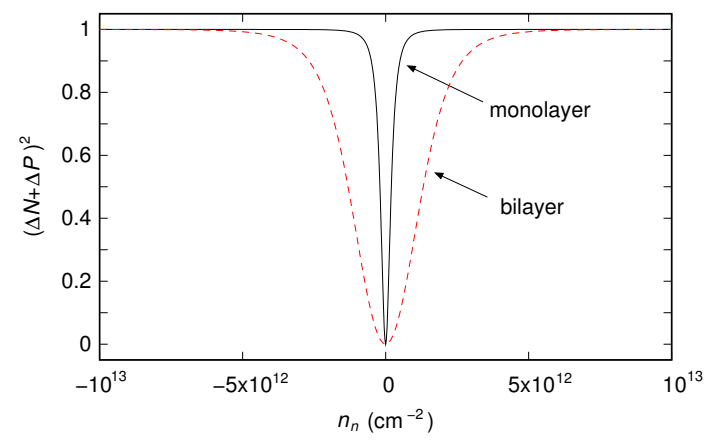

Fig. 3 Behavior of $(\Delta N+\Delta P)^{2}$, as a function of $n_{n}$, for the cases of monolayer and bilayer graphene.
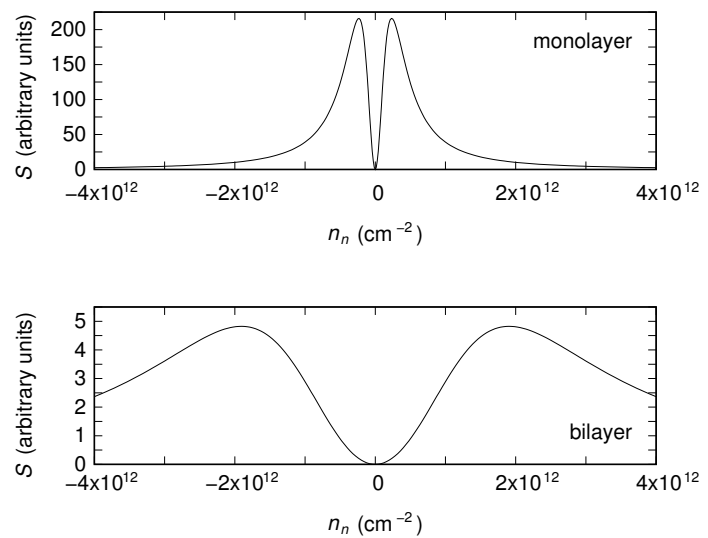

Fig. 4 Behavior of the flicker noise power spectral density $S$ as a function of $n_{n}$, computed with the first method in the text for monolayer (upper panel) and bilayer (lower panel) graphene.
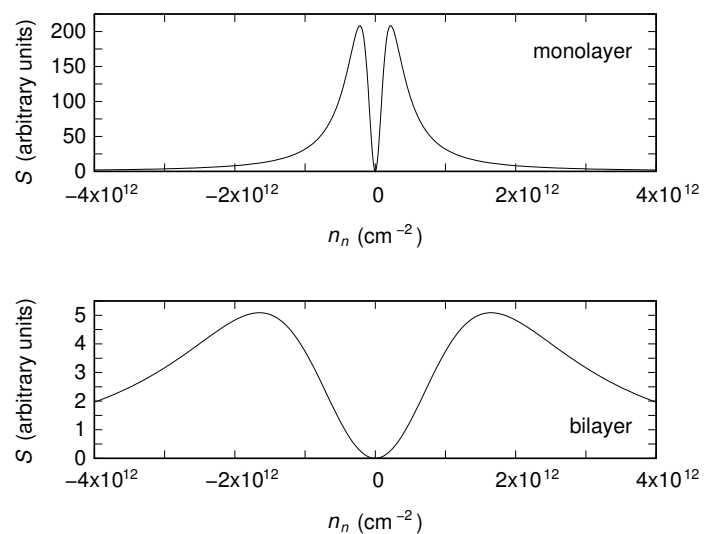

Fig. 5 Behavior of the flicker noise power spectral density $S$ as a function of $n_{n}$, computed with the second method in the text for monolayer (upper panel) and bilayer (lower panel) graphene.

often observed in experiments on bilayer graphene, but only seldom in the case of monolayer graphene (see, e.g. Ref. [2,21]).

We now introduce the fact that the electrostatic potential in graphene is not at all uniform across the sheet, but, rather, it fluctuates, due to the presence of impurities and of defects. This involves that the power spectral density of Eqs. (6) and (11) has to be weighted with the distribution function $P(U)$ 
of the electrostatic potential energy:

$$
\begin{gathered}
\frac{\langle S\rangle}{I^{2}}=\frac{\eta}{A f^{\gamma}} \int\left(\frac{a_{c}}{a n_{c}}\right)^{2} P(U) d U, \\
\frac{\langle S\rangle}{I^{2}}=\frac{\eta^{\prime}}{A f^{\gamma}} \int\left(\frac{\Delta N+\Delta P}{n_{c}}\right)^{2} P(U) d U .
\end{gathered}
$$

\section{Results}

If we assume a Gaussian form for $P(U)$, with a standard deviation $\sigma^{*}$, for monolayer graphene the relatively narrow depression around the Dirac point can be reduced ( $M$ shape) or even disappear $(\Lambda$ shape). This can be seen in Figs. 6 and 7, where we report the averaged flicker noise power spectral density for a few different value of the standard deviation $\sigma^{*}$ obtained from Eqs. (12) and (13), respectively.

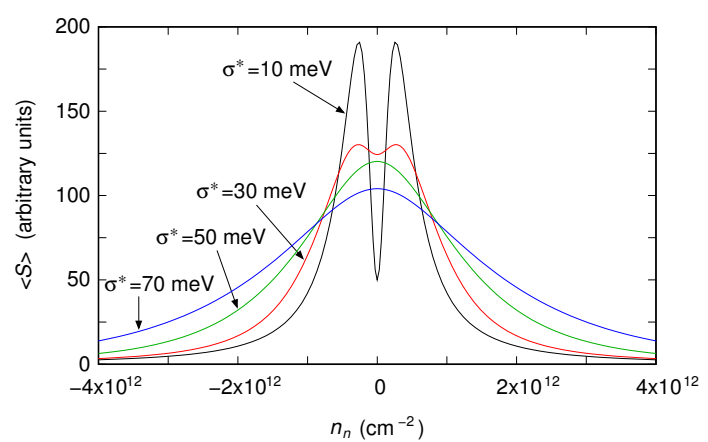

Fig. 6 Behavior, as a function of $n_{n}$, of the flicker noise power spectral density of monolayer graphene averaged over the spatially varying electrostatic potential. The results have been computed using the first method in the text, for four different values of the standard deviation $\sigma^{*}$ of the distribution of the potential energy.

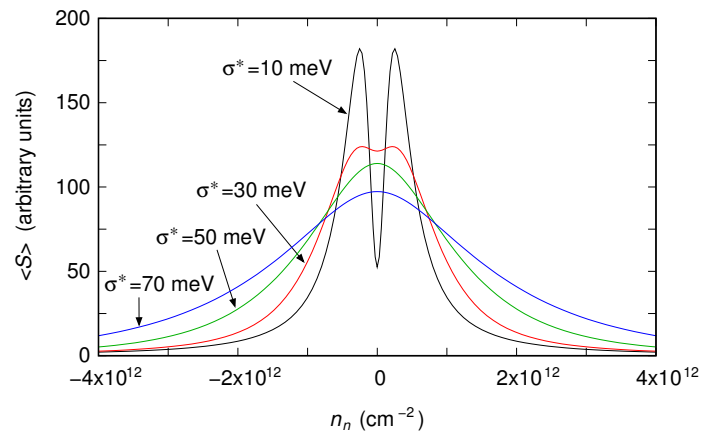

Fig. 7 Behavior, as a function of $n_{n}$, of the flicker noise power spectral density of monolayer graphene averaged over the spatially varying electrostatic potential. The results have been computed using the second method in the text, for four different values of the standard deviation $\sigma^{*}$ of the distribution of the potential energy.

Considering the approach based on the use of the mass-action law for determining the fluctuation $\Delta n_{c}$, we observe that the result is valid as long as the trapping-detrapping events are slow compared with the thermal generation-recombination time constants. At extremely low temperatures such time constants may become very large [22], and in such a case $\Delta n_{c}$ would always be unitary, independent of 
carrier density, thus leading to a disappearance of the minimum of the shot noise power density both for monolayer and bilayer graphene. A hint in this direction can be found, for a monolayer graphene sample that at room temperature exhibits an $M$-shape behavior, in Ref. [21], although similar measurements do not yield an equivalently clear-cut result in Ref. [3].

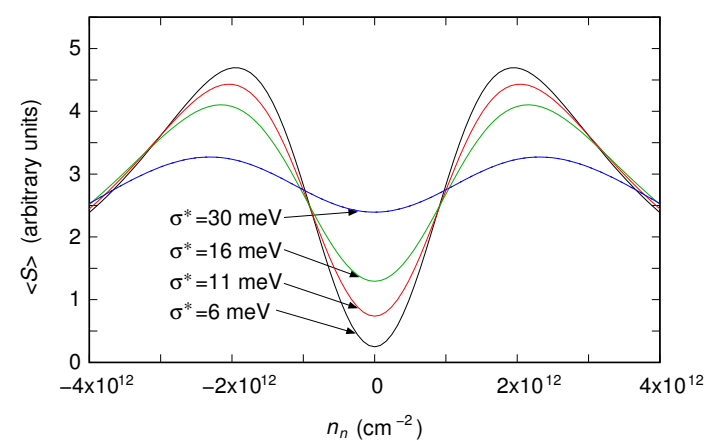

Fig. 8 Behavior, as a function of $n_{n}$, of the flicker noise power spectral density of bilayer graphene averaged over the spatially varying electrostatic potential. The results have been computed using the first method in the text, for four different values of the standard deviation $\sigma^{*}$ of the distribution of the potential energy.

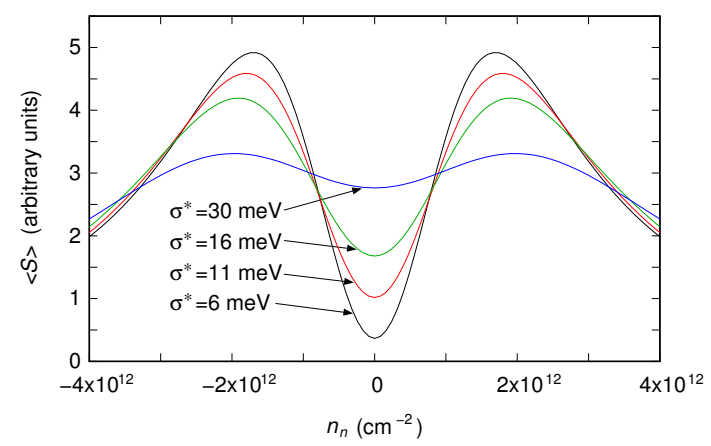

Fig. 9 Behavior, as a function of $n_{n}$, of the flicker noise power spectral density of bilayer graphene averaged over the spatially varying electrostatic potential. The results have been computed using the second method in the text, for four different values of the standard deviation $\sigma^{*}$ of the distribution of the potential energy.

For bilayer graphene we consider smaller values of the standard deviation of the electrostatic potential, because of the larger screening of the effect of random impurities (which are the main source of the fluctuations). A measure of the screening is represented by $\left|\partial U / \partial n_{n}\right|$, which, based on the previous definitions, corresponds to $|1 / a|$. Since in the Dirac point the value of $1 / a$ is about 6 times larger for monolayer graphene than for bilayer graphene (see Fig. 1), we use a similar scaling of the standard deviation. In Figs. 8 and 9 we report the behavior of the flicker noise power spectral density as a function of $n_{n}$ for bilayer graphene, and we notice that in this case the minimum at the Dirac point never disappears, as a result both of the much smoother variation of the density of states of bilayer graphene with respect to what happens for monolayer graphene and of the reduced potential fluctuation.

\section{Conclusions}

We have presented a model for flicker noise in graphene devices that reproduces the experimentally observed behavior in terms of the dependence of the noise power spectral density on carrier density (or, equivalently, gate bias) for monolayer and bilayer graphene. We have shown that the symmetry around 
the $k$ axis of the graphene bandstructure leads to a suppression of flicker noise around the Dirac point (due to the bipolar electron-hole conduction) and that the different observed behaviors can be explained considering a random fluctuation of the electrostatic potential in the graphene sheet. These results may be relevant also for other semiconducting materials characterized by a strong symmetry between electrons and holes: in the case of limited disorder it should be possible to observe a suppression of noise due to carrier trapping phenomena when electron and hole concentrations are equal. In addition, our results hint at a possible change of behavior at very low temperature, due to the much longer generation-recombination times, which could suppress the dependence of $\Delta n_{c}$ on the carrier density, thus prompting for further experimental activity on monolayer and bilayer graphene at sub-10 K temperatures.

Acknowledgements We acknowledge financial support from the European Commission through the FP7 STREP Project GRADE, under Grant 317839.

\section{References}

1. A. A. Balandin, Low-frequency $1 / f$ noise in graphene devices, Nature Nanotechnology 8, 549 (2013).

2. G. Xu, C. M. Torres Jr., Y. Zhang, F. Liu, E. B. Song, M. Wang, Y. Zhou, C. Zeng, and K. L. Wang, Effect of Spatial Charge Inhomogeneity on 1/f Noise Behavior in Graphene, Nano Letters 10, 3312 (2010).

3. Y. Zhang, E. E. Mendez, and X. Du, Mobility-dependent low frequency noise in Graphene field effect transistors, ACS Nano 5, 8124 (2011).

4. S. Rumyantsev, G. Liu, W. Stillman, M. Shur, and A. A. Balandin, Electrical and noise characteristics of graphene field-effect transistors: ambient effects, noise sources and physical mechanisms, J. Phys.: Condens. Matt. 22, 395302 (2010).

5. I. Heller, S. Chatoor, J. Maennik, M. A. G. Zevenbergen, J. B. Oostinga, A. F. Morpurgo, C. Dekker, and S. G. Lemay, Charge Noise in Graphene Transistors, Nano Letters 10, 1563 (2010).

6. A. N. Pal, S. Ghatk, V. Kochat, E. S. Sneha, A. Sampathkumar, A, Raghavan, and A. Ghosh, Microscopic Mechanism of 1/f Noise in Graphene: Role of Energy Band Dispersion, ACS Nano 5, 2075 (2011).

7. F. N. Hooge, $1 / f$ noise is no surface effect, Physics Letters A 29, 139 (1969).

8. Y. Lin and P. Avouris, Strong Suppression of Electrical Noise in Bilayer Graphene Nanodevices, Nano Letters 8, 2119 (2008).

9. B. Pellegrini, Electric charge motion, induced current, energy balance, and noise, Phys. Rev. B 34, 5921 (1986).

10. S. Ramo, Currents Induced by Electron Motion, Proceedings of the IRE 27, 584 (1939).

11. W. Shockley, Currents to Conductors Induced by a Moving Point Charge, J. Appl. Phys. 9, 635 (1938).

12. B. Pellegrini, $1 / f$ noise in graphene, Eur. Phys. J. B 86, 373 (2013).

13. P. Marconcini, M. Macucci, Approximate calculation of the potential profile in a graphene-based device, IET Circ Device Syst 9, 30 (2015).

14. P. Marconcini, M. Macucci, The $k \cdot p$ method and its application to graphene, carbon nanotubes and graphene nanoribbons: the Dirac equation, La Rivista del Nuovo Cimento 34, 489 (2011).

15. D. Logoteta, P. Marconcini, C. Bonati, M. Fagotti, M. Macucci, High-performance solution of the transport problem in a graphene armchair structure with a generic potential, Phys. Rev. E 89, 063309 (2014).

16. E. McCann, Asymmetry gap in the electronic band structure of bilayer graphene, Phys. Rev. B 74, 161403(R) (2006).

17. S. Machlup, Noise in Semiconductors: Spectrum of a Two-Parameter Random Signal, J. Appl. Phys. 25, $341(1954)$

18. A. L. McWhorter, $1 / f$ noise and germanium surface properties in Semiconductor Surface Physics, ed. by R. H. Kingstone, University of Pennsylvania Press, p. 207 (1957).

19. B. Pellegrini, A general model of $1 / f^{\gamma}$ noise, Microel. Reliab. 40, 1775 (2000).

20. T. Fang, A. Konar, H. Xing, D. Jena, Carrier Statistics and quantum capacitance of graphene sheets and ribbons, Appl. Phys. Lett. 91, 092109 (2007).

21. A. A. Kaverzin, A. S. Mayorov, A. Shytov, and D. W. Horsell, Impurities as a source of $1 / f$ noise in graphene, Phys. Rev. B 85, 075435 (2012).

22. F. Rana, P. A. George, J. H. Strait, J. Dawlaty, S. Shivaraman, Carrier recombination and generation rates for intervalley and intravalley phonon scattering in graphene, Phys. Rev. B 79, 115447 (2009). 